\title{
Design and Management of Multi-functional Exclusive Lane for the Integrated Service to Various Vehicles with Priority
}

\author{
Zhiquan Xie $^{\oplus a}$, Hui Jin ${ }^{\odot b}$, Jing Teng ${ }^{\circledR a}$, and Xiaoguang Yang ${ }^{\circledR a}$ \\ ${ }^{a}$ Key Laboratory of Road and Traffic Engineering of the Ministry of Education, Tongji University, Shanghai 201800, China \\ ${ }^{b}$ School of Rail Transportation, Soochow University, Suzhou 215131, China
}

\section{ARTICLE HISTORY}

Received 8 May 2021

Revised 31 July 2021

Accepted 5 September 2021

Published Online 11 November 2021

\section{KEYWORDS}

Multi-functional exclusive lane

Various priority levels

Utility function

HOV critical occupancy

LOV price rate

\begin{abstract}
Priority to emergency vehicles, buses, high occupancy vehicles (HOVs), and priced low occupancy vehicles (LOVs) is critical to their efficiency and reliability, where multi-functional exclusive lane (MFEL) serves as a salient element with independent operation environment. To enhance MFEL utility and balance the demand between prioritized and non-prioritized vehicles, this research proposes an optimization model for MFEL design and management to comprehensively serve the trip modes with various priority levels and distinct operation patterns, considering the delay from lane access, bus stop dwelling, signalized intersections, and ride-sharing. Case study follows to calibrate and validate the proposed model under varying passenger demand and emergency vehicle frequency, finding that MFEL may reduce total travel time in all scenarios especially under high traffic demand. Sensitivity analyses test the effect of road lane count and bus occupancy on MFEL design and management, where the road with more lanes is more flexible to accommodate increased passenger demand without increasing HOV critical occupancy or LOV price rate. Moreover, higher bus occupancy assists in avoiding significant increase of LOV price rate to promote trip equity. This research may lay foundation to MFEL implementation to mitigate traffic congestion and promote transport sustainability.
\end{abstract}

\section{Introduction}

Exclusive lanes have gone a long history to provide specified transport modes independent and prioritized operation environment, so as to offer travelers various trip modes such as environmentfriendly and space-efficient bus or trip-sharing, or to choose to pay the express lane for reduced delay, which implicates exclusive bus lane (EBL), high occupancy vehicle (HOV) lane, and high occupancy toll (HOT) lane, respectively. Though these management lanes are widely advocated over the world, they are faced with serious challenges such as utility rate that questions their warrant. For example, on the road with limited bus routes, EBL is hard to be justified according to the existing standard on bus frequency or hourly ridership (TCRP, 2013). Consequently, HOV and HOT lanes are proposed in sequence, which allow HOVs and priced LOVs to share exclusive lanes with buses. Further, challenged with more urban emergency events, the lane that secures the passage of fire-engines, ambulance, and engineering emergency vehicles is also critical to society and economy development (Nellore et al., 2016). Therefore, there is urgent need to develop multi-functional exclusive lane (MFEL) for the joint use by buses, HOVs, priced LOVs, and emergency vehicles on the urban road, utility of which calls for the comprehensive exploration on MFEL design and management to address the different operation patterns of various prioritized trip modes.

EBL dates back to around 1940s in Chicago, which develops quickly in 1970s to enhance transit efficiency and reliability, and has accumulated more than $300 \mathrm{~km}$ in Beijing, China (Xu et al., 2013). However, EBL suitability and utility is still open to debate on the determinants of bus volume (Wu et al., 2018), passengers (Abdelfatah and Abdulwahid, 2017), and road conditions (Liu et al., 2015). For example, Wu et al. (2017) incorporate the uncertainty of bus operation time and dwell time at stop into stochastic programming model for EBL design. With VISSIM simulation, it is found that when traffic saturation exceeds 0.8, EBL can significantly reduce bus delay at the cost of increasing car delay. 
To avoid the negative effect of EBL on cars and to enhance EBL utility, dynamic bus lanes (DBL) have been proposed, where cars are forbidden only when there come buses. With cell automata (CA) model, DBL mechanism is investigated for the optimal design to enhance road utility, which is extended by $\mathrm{Wu}$ et al. (2018) for basic DBL parameters, such as clearance distance, traffic saturation, and bus headway (Liu et al., 2015). However, Sweden DBL pre-study finds that cars changing onto and out of DBL may pose significant influence on traffic efficiency and safety (Olstam et al., 2015).

In contrast, high occupancy vehicle (HOV) lane provides a more reliable expressway (Yang, 1998). But still, these lanes are accused of low utility level, and purely loosening the criteria of vehicle occupancy cannot solve this problem (Kwon and Varaiya, 2008; Brown and Aicp, 2008).To this end, HOT lane is proposed to allow LOVs when they pay a specified fare (Jang et al., 2014), which has been recently explored regarding design, simulation, implementation, and evaluation (Lombardi et al., 2021). Empirical research has pointed that travelers use HOT lanes on an infrequent basis (Burris and Stockton, 2004), and the scientific fare rate is critical to HOT lane success. Combining HOVs and LOVs, a bilevel framework is structured to explore LOV toll rate based on the relationship between vehicle demand and trip time (Jang et al., 2014). Empirical research on the conversion of HOV into variably priced HOT lanes in Miami, Minneapolis, and Atlanta finds that bus travel time saving and ridership increase are $17 \mathrm{~min}$ and $57 \%, 4.5 \mathrm{~min}$ and $13 \%$, and $5 \mathrm{~min}$ and $11 \%$, respectively (Pessaro et al., 2013). Theoretical research follows to find that maximum revenue is achieved by keeping the toll facility at capacity with no queues for as long as possible (Laval et al., 2015). Tan and Gao (2018), targeting at HOT lane with multiple entrances and exits, establish a mixed integer linear programming model to coordinate dynamic fare schemes with varying OD pairs. The access and exit locations along the HOT lanes are also explored, motivated by HOT lane accessibility (Zang, 2021) and vehicular speed difference between HOT and general purpose lanes (GPLs) (Zitzow-Childs, 2017). It is found that HOT lanes with entrance close to city center attract more users while those near city edge help secure operation efficiency. Parking space constraint has also been included into modal choice of commuting over management lanes (Xiao et al., 2016; Xiao et al., 2019). Faced with social equity problem from HOT, investigation on the willingness to pay HOT lanes has found that high-income travelers benefit most, while low-income travelers are least advantaged. To this end, the tradable credit scheme, a revenue-neutral measure, has been proposed for managed lanes so as to avoid public resistance from paying a toll (Zang, 2021).

Further, as more transit agencies seek the knowledge to take advantage of such new infrastructure opportunity, more efforts have been made on the impact of HOT lanes to transit. Recent research identifies that the salient elements of HOT lanes for transit agencies include bus service provision, park and ride provision, transit ridership, as well as transit subsidy (Newmark, 2014). Survey towards university students shows that bus punctuality in addition to trip time saving increases the willingness to use bus for commuting on HOT lanes (Rossi, 2019). As most operators of current and planned express toll lanes appreciate the synergy between freer-flowing lanes and better express bus service, Maryland DOT has unveiled its plans for express bus service on the managed lanes currently being procured for the Maryland (Poole, 2020).

Table 1. Notation of the Proposed Model

\begin{tabular}{|c|c|}
\hline Notation & Explanations \\
\hline \multicolumn{2}{|l|}{ Indices } \\
\hline$m$ & Index of trip mode \\
\hline \multicolumn{2}{|l|}{ Sets } \\
\hline$\{E, B, H, L, G\}$ & $\begin{array}{l}\text { Vehicle types of emergency vehicles, buses, HOVs, priced } \\
\text { LOVs, and general vehicles }\end{array}$ \\
\hline \multicolumn{2}{|l|}{ Parameters } \\
\hline$\mu_{m}$ & Equivalent car coefficient of trip mode $m$ \\
\hline$O_{m}(m \neq H)$ & Vehicle occupancy of trip mode $m$ (except HOV) \\
\hline$N$ & Total count of travelers \\
\hline$a, b, c$ & Utility coefficients \\
\hline$L_{0}$ & Total number of road lanes \\
\hline$P$ & Capacity of each lane, veh/h/ln \\
\hline$D$ & Trip length, km \\
\hline$v_{f}$ & Free-flow speed, $\mathrm{km} / \mathrm{h}$ \\
\hline$\beta, \alpha$ & Coefficients of BRP function \\
\hline$d_{m_{-} a c}, d_{m_{-} r s}$ & $\begin{array}{l}\text { Delay from lane access and ride-sharing of mode } m \text { per trip } \\
\text { distance, respectively, } \mathrm{h} / \mathrm{km}\end{array}$ \\
\hline$P_{B}, p_{v}$ & $\begin{array}{l}\text { Priority probability of buses and } \mathrm{HOV} / \mathrm{LOVs} \text { at intersec- } \\
\text { tions, respectively }\end{array}$ \\
\hline$\theta$ & $\begin{array}{l}\text { Additional delay caused by the absolute priority of emer- } \\
\text { gency vehicles, } \mathrm{h} / \mathrm{veh}\end{array}$ \\
\hline$C$ & Signal cycle length, $\mathrm{s}$ \\
\hline$u$ & Green ratio \\
\hline$M$ & Randomly large constant \\
\hline$s$ & Stop density per km \\
\hline$d_{s w}$ & Bus dwelling time, $\mathrm{s}$ \\
\hline$p r_{b}, p r_{f}, p r_{k}$ & Price of bus ticket, fuel cost, and parking cost, respectively, RMB \\
\hline$W_{m}$ & Weight of trip mode $m$ \\
\hline \multicolumn{2}{|c|}{ Intermediate variables } \\
\hline$n$ & Total number of equivalent cars \\
\hline$n_{m}(m \neq G)$ & Vehicle count of mode $m$ (except GV) \\
\hline$U_{m}$ & Utility of trip mode $m$ \\
\hline$t t_{m}, p r_{m}$ & $\begin{array}{l}\text { Total travel time (s) and trip cost (RMB) of mode } m \text {, respec- } \\
\text { tively }\end{array}$ \\
\hline$t_{m}, d_{m}$ & $\begin{array}{l}\text { Total running time and delay of mode } m \text { per trip distance, } \\
\text { respectively, } \mathrm{h} / \mathrm{km}\end{array}$ \\
\hline$d_{m_{-} p p}, d_{m_{-} i t}$ & $\begin{array}{l}\text { Delay from bus stop dwelling and intersections of mode } m \\
\text { per trip distance, respectively, } \mathrm{h} / \mathrm{km}\end{array}$ \\
\hline$x_{m}$ & $\begin{array}{l}\text { Ratio of vehicle arrival to intersection capacity of the lane } \\
\text { that mode } m \text { is on }\end{array}$ \\
\hline \multicolumn{2}{|c|}{ Decision variables } \\
\hline$n_{p}, n_{G}$ & Equivalent vehicle count on MFEL and on GPL, respectively \\
\hline$e$ & A binary variable indicating whether MFEL is adopted or not \\
\hline$L$ & Number of MFELs on the road \\
\hline$O_{H}$ & HOV critical occupancy \\
\hline$p r_{L}$ & $\mathrm{LOV}$ price rate per trip distance, $\mathrm{RMB} / \mathrm{km}$ \\
\hline
\end{tabular}


In summary, the combination of buses, HOVs, and priced LOVs has been increasingly researched, which generally presets the minimum HOV occupancy and pre-deploys bus service on one managed lane, instead of establishing a comprehensive framework for the integrated design and management of multifunctional exclusive lanes (MFEL) so as to minimize total travel time against site-specific traffic conditions.

The following section summarizes the notation in the research. Section 3 establishes MFEL mathematical modeling, solution to and case study of which is developed in Section 4 and 5 , respectively. Section 6 gives the brief conclusions.

\section{Notation}

Prioritized vehicles allowed on the MFEL as well as general vehicles (GVs) on the GPLs will be separately represented to reflect their distinct travel patterns and priority levels. Based on trip mode choice, mode utility of buses, HOVs, priced LOVs, and GVs is structured with respect to trip time and cost so as to split trip demand, where emergency vehicle frequency is exogenously determined. To facilitate model presentation, notation used hereafter is summarized in Table 1.

\section{MFEL Mathematical Modeling}

Figure 1 shows the mechanism of MFEL serving emergency vehicles, buses, HOVs, and LOVs, but not GVs. Fig. 2 gives the framework of MFEL design and management, where road lanes may serve as MFEL or GPL and trip demand can be distributed to the modes with or without priority. Prioritized trip modes include emergency vehicles, buses, HOVs, and priced LOVs, where HOVs are characterized with critical occupancy, and LOVs correspond to price rate, both influencing trip mode utility that determines travelers' modal choice. Traffic volume can be represented with

$$
n=n_{p}=n_{G}=\left(\mu_{E} n_{E}+\mu_{B} n_{B}+\mu_{H} n_{H}+\mu_{L} n_{L}\right)+n_{G},
$$

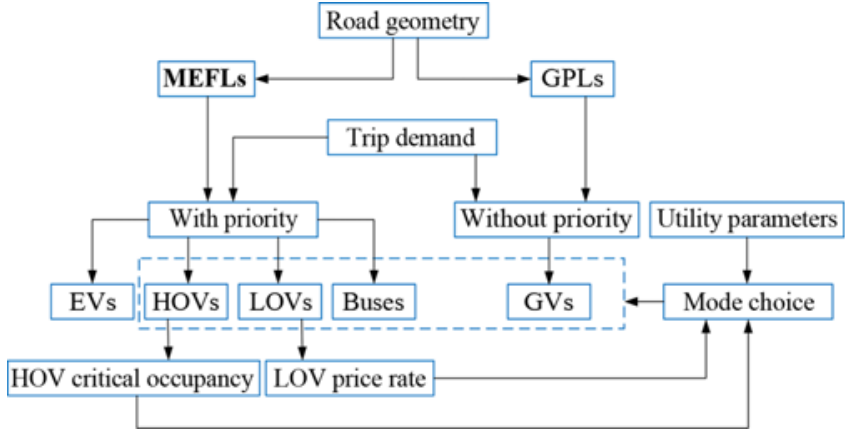

Fig. 2. Framework of MFEL Design and Management

where $n$ represents the total number of equivalent cars, $n_{p}$ and $n_{G}$ means equivalent vehicles on MFEL and GPL, respectively; $\mu_{E}$, $\mu_{B}, \mu_{H}$, and $\mu_{L}$ are the equivalent coefficient of emergency vehicles $\left(n_{E}\right)$, buses $\left(n_{B}\right)$, HOVs $\left(n_{H}\right)$, and priced LOVs $\left(n_{L}\right)$. With mode choice model, vehicle count is given by

$$
n_{m}=\frac{\exp \left(-U_{m}\right)}{\sum_{m} \exp \left(-U_{m}\right)} \times \frac{N}{O_{m}} \quad \forall m \in\{B, H, L, G\},
$$

where $U_{m}$ and $O_{m}$ represent the utility and vehicle occupancy of trip mode $m$, respectively; $N$ is the total count of travelers. Despite significant stochasticity of trip demand and varying trip mode preference (Tirkolaee and Hadian, 2019), deterministic model is employed in this paper for simplification and to focus on the relationship among trip demand, road resource, and MFEL design/management. Utility $U_{m}$ is given by (Nuzzolo and Comi, 2016)

$$
U_{m}=a+b \times t t_{m}+c \times p r_{m} \quad \forall m \in\{B, H, L, G\},
$$

where $a, b$ and $c$ are the utility coefficients, and $t t_{m}$ and $p r_{m}$ refer to the total travel time and trip cost of mode $m$. Parameter $t t_{m}$ is given by

$$
t t_{m}=\sum_{m} D \times\left(t_{m}+d_{m}\right) \quad \forall m \in\{B, H, L, G\},
$$

where $D$ is average trip distance, $t_{m}$ and $d_{m}$ are the running time

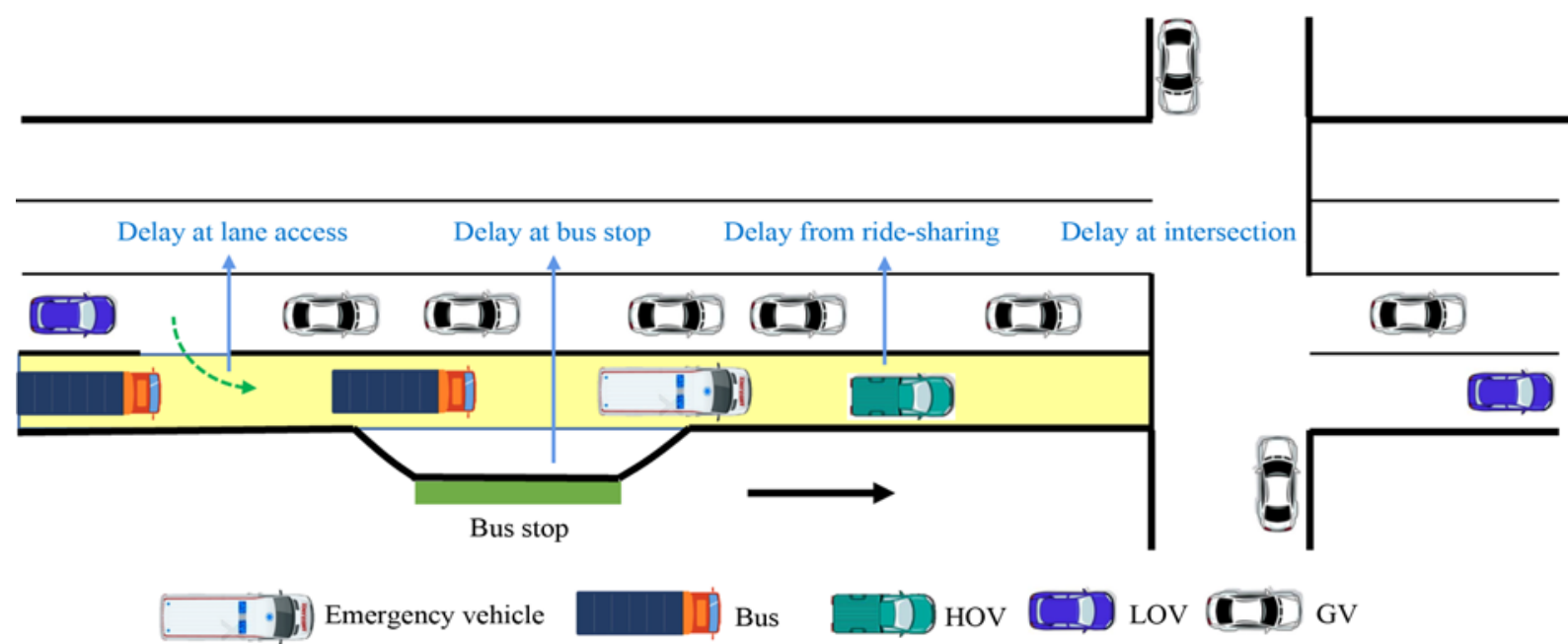

Fig. 1. Illustration of MFEL Mechanism 
and delay of mode $m$ per distance, respectively, which are given by

$$
t_{m}=\left\{\begin{array}{c}
\max \left\{\left[1+\beta\left(\frac{n_{G}}{\left(L_{0}-L\right) P}\right)^{\alpha}\right] \frac{e}{v_{f}},\left[1+\beta\left(\frac{n}{L_{0} P}\right)^{\alpha}\right] \times \frac{(1-e)}{v_{f}}\right\} m=G \\
\max \left\{\left[1+\beta\left(\frac{\sum_{m \neq G} n_{m}}{(L+1-e) P}\right)^{\alpha}\right] \frac{e}{v_{f}},\left[1+\beta\left(\frac{n}{L_{0} P}\right)^{\alpha}\right] \times \frac{(1-e)}{v_{f}}\right\} \\
m \in\{E, B, H, L\}
\end{array}\right.
$$

$L \leq M \times e$,

$0 \leq O_{H}-1 \leq M \times e$,

$0 \leq p r_{L} \leq M \times e$,

$$
\begin{gathered}
d_{m}=\left[d_{m_{-} a c}, d_{m_{-} s p}, d_{m_{-} i t}, d_{m_{-} r s}\right] \times\left[(0,1,1,1)^{T} \times(1-e)+\overrightarrow{S_{m}}\right] \\
m \in\{E, B, H, L, G\}
\end{gathered}
$$

$$
\overrightarrow{s_{m}}=\left[s_{a c}, s_{t p}, s_{i t}, s_{r s}\right]_{m}^{T}= \begin{cases}(0,0,0,0)^{T} & m=E \\ \left(0,1, p_{B}, 1\right)^{T} \times\left(1+\theta n_{E}\right) e & m=B \\ \left(0,1, p_{V}, 1\right)^{T} \times\left(1+\theta n_{E}\right) e & m=H . \\ \left(0,1, p_{V}, 1\right)^{T} \times\left(1+\theta n_{E}\right) e & m=L \\ (1,1,1,1)^{T} \times\left(1+\theta n_{E}\right) e & m=G\end{cases}
$$

Equation (5) describes the travel time of GVs and prioritized vehicles, respectively (Saberi and Figliozzi, 2011). In each relationship, the first term refers to the travel time with MFEL, while the second without, as indicated by the binary variable $e$. That is, if MFEL is adopted, then $e=1$; otherwise, $e=0$. Parameter $\beta$ and $\alpha$ are the coefficients of BRP function, $L_{0}$ is the total number of lanes on the road, $L$ is the number of MFELs, $P$ is lane capacity, and $v_{f}$ is free-flow speed. Eqs. (6) to (8) give the relationship between the binary variable $e$ and MFEL count, HOV critical occupancy, and LOV price rate, with $M$ being randomly large constant. That is, when not setting MFEL (i.e., $e$ $=0$ ), constraints (6) to (8) are tightened to make $L=0, O_{H}=1$, and LOV price rate $p r_{L}=0$. Eq. (9) gives the main delay that vehicles undergo during a trip (Lombardi and Picado-Santos, 2021), where $d_{m_{-} a c}, d_{m_{-} s p}, d_{m_{-} i t}$, and $d_{m_{-} r s}$ refer to the delay from lane access, bus stop dwelling, signalized intersections, and ridesharing of mode $m$, respectively; $\overrightarrow{s_{m}}$ represents the priority level of mode $m$. Eq. (10) formulates the priority vector of trip mode $m$ with respect to the four kinds of delay mentioned above: emergency vehicle enjoys full priority and is free of all kinds of delay, buses are prioritized fully at access and with probability $\left(1-p_{B}\right)$ at inter section, while HOVs and LOVs are prioritized with probability $\left(1-p_{V}\right)$ at intersections (Zhou et al., 2020). The term $1+\theta n_{E}$ of the last four equations indicates the additional delay caused by the absolute priority of emergency vehicles, with $\theta$ being the delay coefficient (Boudhrioua and Shatanawi, 2019). Assuming one intersection per kilometre along urban corridor, intersection delay $d_{m \_ \text {it }}$ on each lane is given by (Akcelik,
1988)

$$
d_{m_{-} i t}=\frac{C(1-u)^{2}}{3600 \times 2\left(1-u x_{m}\right)}+\frac{225 x_{m}^{2}}{3600}\left[\left(x_{m}-1\right)+\sqrt{\left(x_{m}-1\right)^{2}+4 x_{m} / 125}\right],
$$

where the first and second term refer to average delay from uniform and random vehicle arrival. Parameter $C$ is signal cycle, $u$ is green ratio, and $x_{m}$ is the ratio of vehicle arrival to intersection capacity on the lane where vehicles of mode $m$ run. Bus stop delay is given by

$$
d_{s p}=s \times d_{s w}+0.5 \frac{3600}{n_{B}} / D,
$$

which is the sum of bus dwelling time and bus waiting time, where the former is the product of stop density $(S)$ and average stop dwelling time $\left(d_{s w}\right)$, and the latter is half bus headway (i.e., $0.5 \frac{3600}{n_{B}}$ ) averaged over trip distance (i.e., D) (Esfeh et al., 2020). Trip cost of Eq. (3) is given by

$$
p r_{m}=\left\{\begin{array}{ll}
p r_{b} & m=B \\
\left(p r_{f}+p r_{k}\right) / O_{H} & m=H \\
\left(p r_{f}+p r_{k}+p r_{L} \times D\right) / O_{H} & m=L \\
\left(p r_{f}+p r_{k}\right) / O_{G} & m=G
\end{array},\right.
$$

where parameters $p r_{b}, p r_{f}$, and $p r_{k}$ refer to the price with respect to bus ticket, fuel cost, and parking cost per trip, respectively, while $p r_{L}$ represents LOV price rate.

Optimization object of MFEL design and management is structured with

$$
\min z=\sum_{m} w_{m} \times n_{m} \times t_{m}
$$

which minimizes the total weighted vehicular delay of each mode. Parameter $w_{m}$ is the weight of trip mode $m$, equal to vehicle occupancy of buses, HOVs, LOVs, and GVs, while the weight of emergency vehicles is exogenously set. Therefore, optimal MFEL design and management is modeled with object of Eq. (14) subjected to Eqs. (1) to (13).

\section{Solution Method}

The problem at hand is a mixed integer non-linear programming model with non-linear constraints with respect to the variables of whether MFEL is set, MFEL count, HOV critical occupancy, LOV price rate, and vehicle count of various trip modes. Specifically, this model is characterized with 13 constraints and 53 variables, among which 6 are decision variables: 1) one binary variable $e$ that indicates whether MFEL is set, 2) four integer variables of critical occupancy $O_{H}$, MFEL count $L$, and vehicle count on the MFEL or GLP; and 3) one variable $p r_{L}$ with one decimal place ranging between 0 and acceptable paying rate. It is noted that, though it is better to take LOV price rate as a continuous variable to be solved in the optimization programming, it will make the non- 


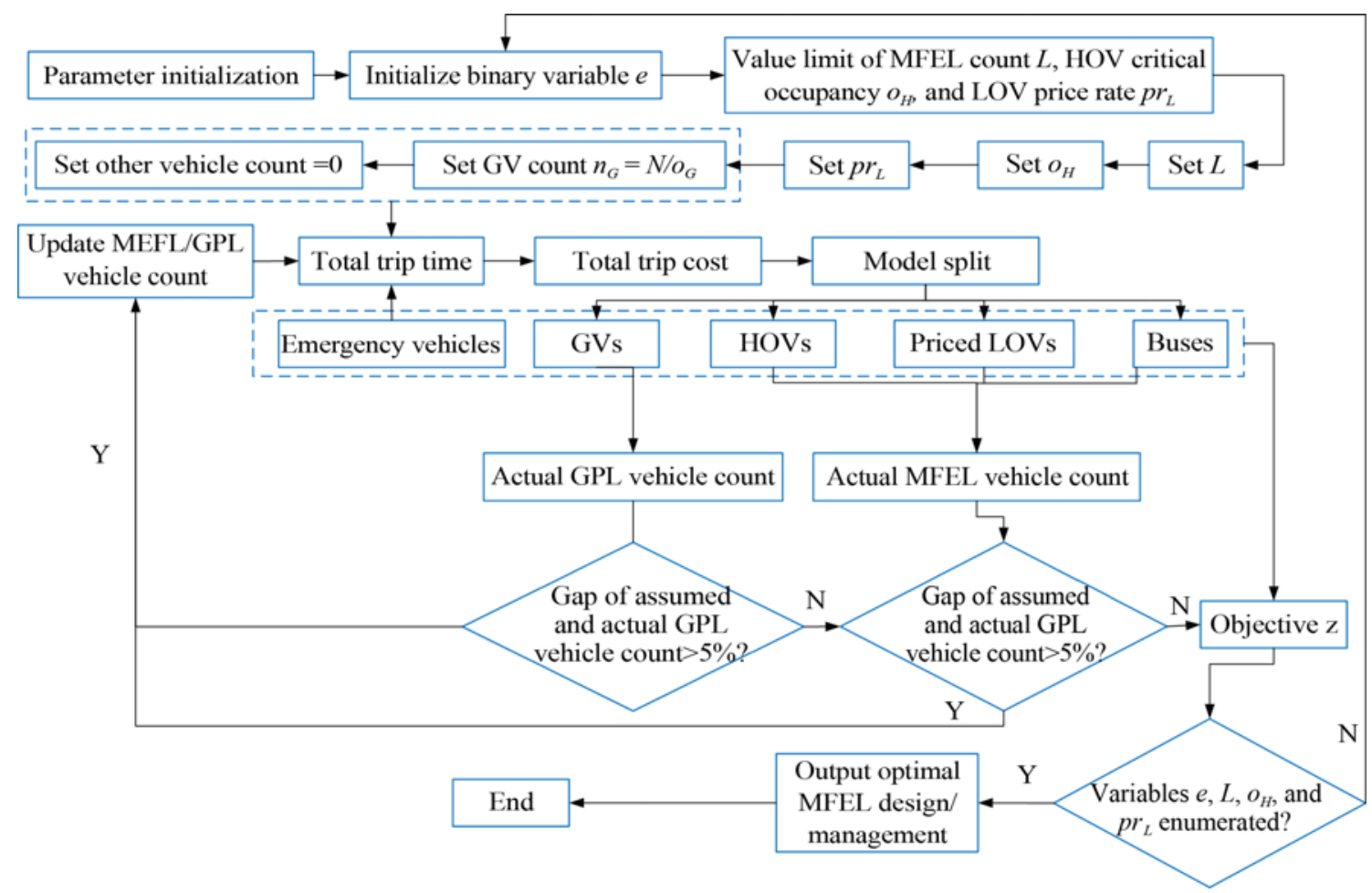

Fig. 3. Framework of Enumeration Algorithm

linear model difficult to be solved. Moreover, when adopting the proposed scheme in practice, LOV price rate is always rounded to be with one decimal point for easy billing, the same as the price rate by Didi and taxi service in China. Therefore, we treated LOV price rate as a discrete variable to make the model easy to be solved, result from which have the acceptable accuracy in the practice. Given the limited range of variables, enumeration is adopted to solve the proposed model for the optimal MFEL design and management.

Figure 3 shows the framework of the model solution algorithm. With initialization, the decision whether MFEL is set is first made, setting the boundary for MFEL count, HOV critical occupancy, and LOV price rate. Specifically, not setting MFEL implicates MFEL count being 0 , HOV critical occupancy being 1 , and LOV price rate being 0 , while MFEL adoption allows MFEL count no larger than total lane count minus 1, HOV critical occupancy between 2 and passenger capacity, and LOV price rate between 0.1 and acceptable paying rate. After deciding MFEL design and management rules, GV count is initialized equal to the total demand divided by GV occupancy, which minimizes GV utility and two cases are discussed: 1) with MFEL, deteriorated GV efficiency returns over-estimation of prioritized vehicle count that lowers MFEL traffic efficiency, and the followed iteration with updated vehicle component gives excessive GVs but to a lesser degree; 2) without MFEL, too many GVs and no buses will deteriorate traffic conditions to narrow the gap between GVs and buses, returning too many buses than expected and enhancing traffic efficiency that overestimates GV share, also to a lesser degree than initialization.
Thus it is concluded that trip mode share converges to the expected value under specified MFEL design and management rules as iteration goes on, where the algorithm stops when the gap between pre-set and returned vehicle count is no larger than $5 \%$.

\section{Case Study and Sensitivity Analyses}

In this section, the proposed model is first calibrated and validated with an experimental scenario. Then sensitivity analyses follow to identify the impact of road lanes and bus occupancy on MFEL design and management.

\subsection{Experimental Design and Model Validation}

The proposed model is tested in the experimental scenario as shown in Fig. 4 with four lanes on the target direction, where traffic experiences delay from MFEL access, bus stop, signalized intersection, as well as ride-sharing. Parameters of the model are summarized in Table 2 , with passenger demand varying between 1,000 and $6,000 \mathrm{psg} / \mathrm{h}$ and emergency vehicles between 0 and 2 $\mathrm{veh} / \mathrm{h}$. Lane capacity is assumed $1,200 \mathrm{veh} / \mathrm{h} / \mathrm{ln}$, free-flow speed is set $60 \mathrm{~km} / \mathrm{h}$, and weight is 1,000 of emergency vehicle travel time relative to passenger delay of other vehicles. Critical HOV occupancy ranges between 2 and $6 \mathrm{psg} / \mathrm{veh}$, and LOV price rate is limited between 0.1 and $2 \mathrm{RMB} / \mathrm{km}$ with interval being 0.1 . That is, LOV price rate can be chosen among $0.1,0.2,0.3, \ldots$, and $2.0 \mathrm{RMB} / \mathrm{km}$.

Figure 5 shows the scheme from the proposed model with MFEL count, MFEL saturation, and GPL saturation, which are 


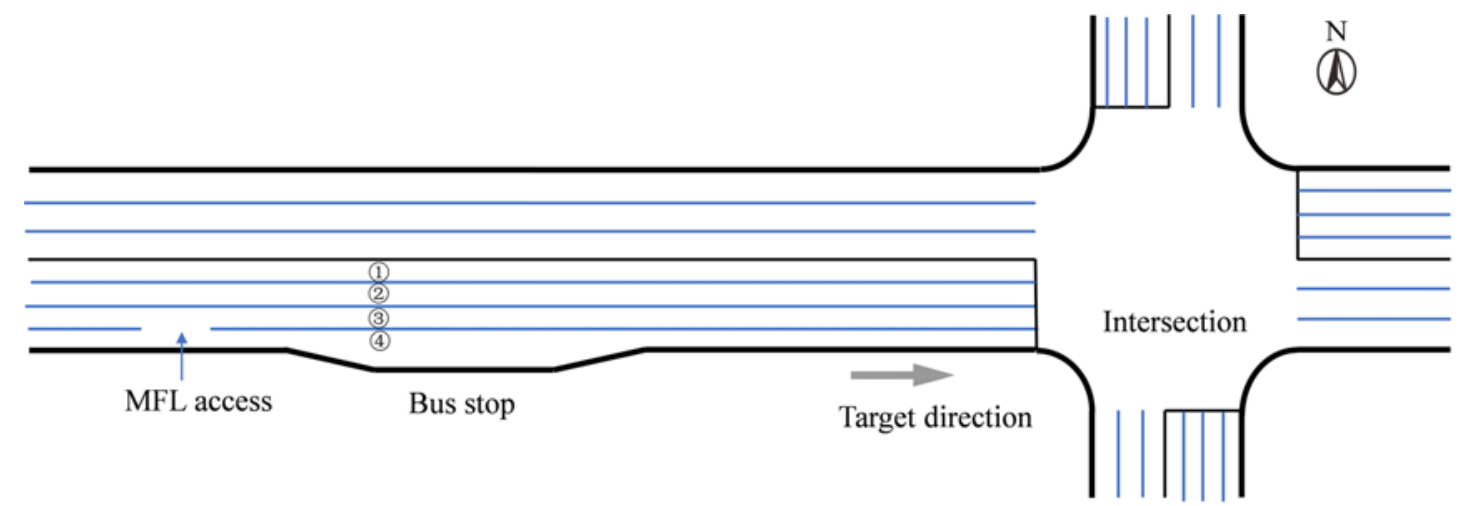

Fig. 4. MFEL Experiment Scenario

Table 2. Parameter Values of the Proposed Model

\begin{tabular}{llllll}
\hline Parameter & Value & Parameter & Value & Parameter & Value \\
\hline$\mu_{E}, \mu_{B}$ & 2 & $v_{f}$ & $60 \mathrm{~km} / \mathrm{h}$ & $M$ & 10,000 \\
$\mu_{H}, \mu_{L}, \mu_{G}$ & 1 & $\theta$ & $20 \mathrm{~s} / \mathrm{veh}$ & $D$ & $10 \mathrm{~km}$ \\
$a$ & 0 & $C$ & $100 \mathrm{~s}$ & $p r_{b}$ & $0.25 \mathrm{RMB} / \mathrm{km}$ \\
$b$ & 30 & $u$ & 0.4 & $p r_{f}$ & $0.75 \mathrm{RMB} / \mathrm{km}$ \\
$c$ & 1 & $O_{B}$ & $45 \mathrm{psg} / \mathrm{veh}$ & $p r_{k}$ & $1.25 \mathrm{RMB} / \mathrm{km}$ \\
$\alpha$ & 4 & $O_{L}, O_{G}$ & $1 \mathrm{psg} / \mathrm{veh}$ & $p_{B}$ & 0.6 \\
$\beta$ & 0.15 & $S$ & $2 \mathrm{per} \mathrm{km}$ & $p_{h}$ & 0.5 \\
$L_{0}$ & 4 & $d_{a c}$ & $20 \mathrm{~s}$ & $w_{E}$ & 1,000 \\
$P$ & $1,200 \mathrm{veh} / \mathrm{h} / \mathrm{ln}$ & $d_{s w}$ & $30 \mathrm{~s}$ & & \\
\hline
\end{tabular}

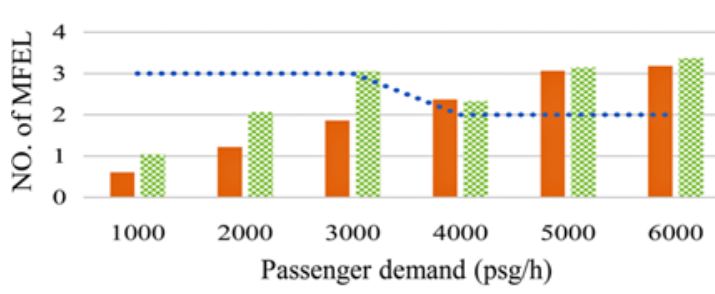

(a)
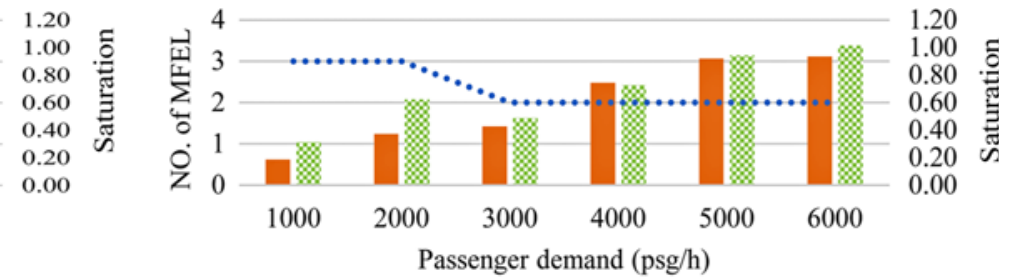

(b)

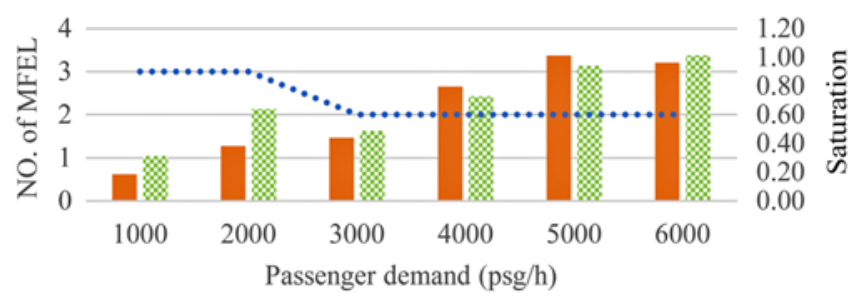

(c)

S-MFEL $\quad \sec s-G P L \quad$........ MFEL

Fig. 5. MFEL Scheme from the Proposed Model with Lane Saturation: (a) Emergency $=0$ veh/h, (b) Emergency $=1$ veh/h, (c) Emergency $=2$ veh/h

represented with MFEL, S-MFEL, S-GPL, respectively. It is observed that the proposed model adopts MFEL regardless of passenger demand levels and emergency vehicle frequency. Specifically, more lanes are set MFEL when passenger demand is relatively low, for example, 3 lanes serve as MFEL when passenger volume is not higher than $2,000 \mathrm{psg} / \mathrm{h}$; while only 2 lanes are MFEL under higher passenger demand. This can be explained that, when faced with higher passenger demand, more cars run on the road to require more lanes, so as to avoid undue delay; while lower passenger demand is free of the challenge of over-saturation and more resource is assigned to prioritized modes to secure their efficiency. Moreover, MFEL saturation is basically lower than that of GPL, and both MFEL and GPL manage to avoid traffic oversaturation. Thus the proposed scheme is 
validated to well balance trip demand with and without priority. Such result improves previous research that simplifies trip delay (e.g., from carpooling or bus dwelling) and the relationship between trip time and vehicle flow, concluding that more exclusive lanes only brings marginal increased total cost reduction under increased trip demand (Yang, 1998; Tan and Gao, 2018).

Figure 6 shows the HOV critical occupancy and LOV price rate on MFEL by the proposed model. It is observed that, under varying frequency of emergency vehicles, HOV critical occupancy does not change, and LOV price rate differs only slightly when passenger demand is $6,000 \mathrm{psg} / \mathrm{h}$. Thus accommodating emergency vehicles does not essentially change MFEL management rules. In other word, MFEL may support the building of urban life line without changing its design or management rules. Further, with respect to the influence of passenger demand, HOV critical occupancy does not increase until passenger demand is high up

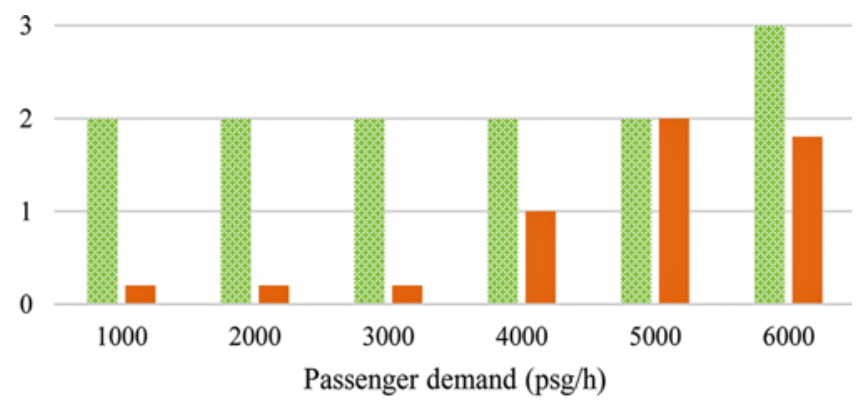

(a)

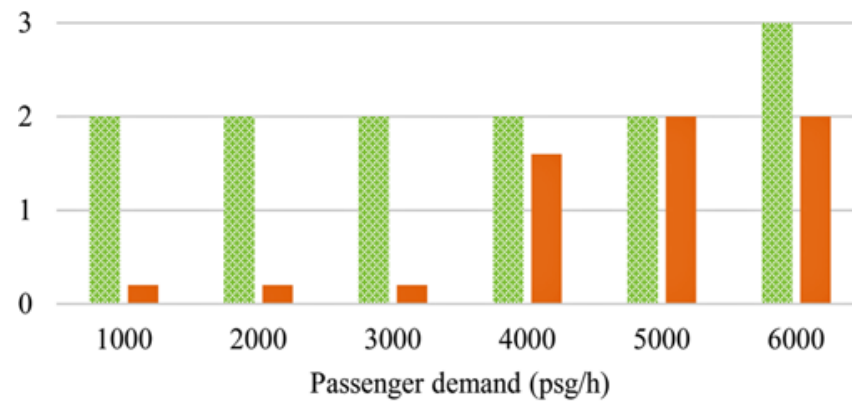

(b)

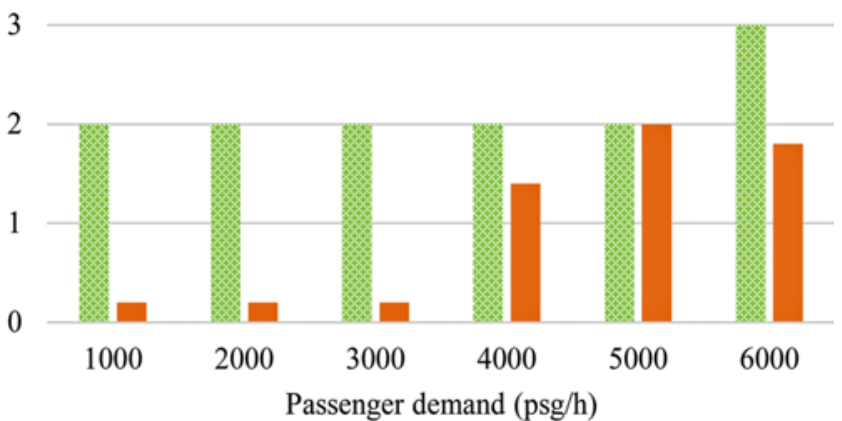

(c)

SOV critical occupancy $=$ LOV price rate

Fig. 6. HOV Critical Occupancy and LOV Price Rate from the Proposed Model: (a) Emergency $=0 \mathrm{veh} / \mathrm{h}$, (b) Emergency $=1 \mathrm{veh} / \mathrm{h}$, (c) Emergency $=2 \mathrm{veh} / \mathrm{h}$

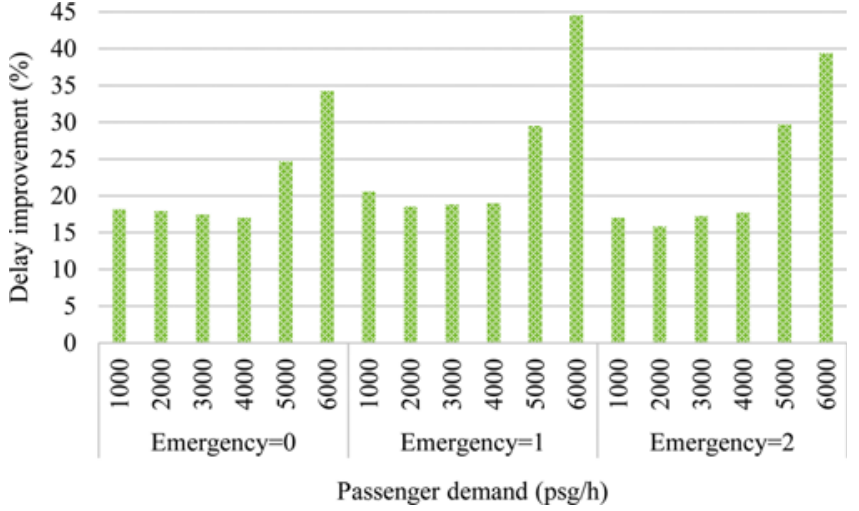

Fig. 7. Improvement of Traffic Delay with MFEL under Varying Passenger Demand

to $6,000 \mathrm{psg} / \mathrm{h}$, where HOVs are required to carry more travelers to enhance road resource utility. Similarly, LOV price rate stays low when passenger demand does not exceed 3,000 psg/h. Thus it is concluded that intense trip demand corresponds to higher HOV occupancy and LOV price rate, i.e., stricter MFEL management criteria, to balance the demand on MFEL and GPL as given by the saturation rate in Fig. 5. Such finding extends the empirical research concluding that HOV critical occupancy itself cannot enhance road utility (Brown and Aicp, 2008; Kwon and Varaiya, 2008), providing insights to the optimal combination of HOV occupancy and LOV price rate.

Figure 7 demonstrates that the proposed MFEL management scheme reduces total traffic delay whatever passenger demand level is, consistent to the finding in previous research (Yang, 1998; Tan and Gao, 2018) but to a greater degree. Moreover, traffic delay decreases more in the cases with higher passenger demand. For example, when passenger demand is $6,000 \mathrm{psg} / \mathrm{h}$ or higher, delay reduction exceeds $30 \%$. Thus it is validated that the simultaneous modeling of HOV critical occupancy and LOV price rate strengthens MFEL advantage to better serve varying trip demand for minimum total trip time, instead of pre-setting HOV occupancy (Rossi and Melissa, 2019).

\subsection{Sensitivity Analysis}

\subsubsection{Effect of Road Lanes}

Figures 8 and 9 show MFEL design and management for the road with 2 or 3 lanes. It is observed that there is always one MFEL for 2-lane road and two MFELs for 3-lane road, regardless of passenger demand and emergency vehicle frequency. Combining with the results from Fig. 7 for the road with 4 lanes, it is concluded that MFEL management can be more complex for the corridor with more lanes to carefully balance road resource for prioritized and non-prioritized trip modes. Further, with respect to HOV critical occupancy, the road with 2 lanes requires at least 3 passengers per vehicle when passenger demand is high up to $3,000 \mathrm{psg} / \mathrm{h}$, while the road with 3 lanes always allow vehicles carrying 2 passengers. Combining the result from Fig. 6 for 4lane road, it is concluded that the road with more than 2 lanes can 


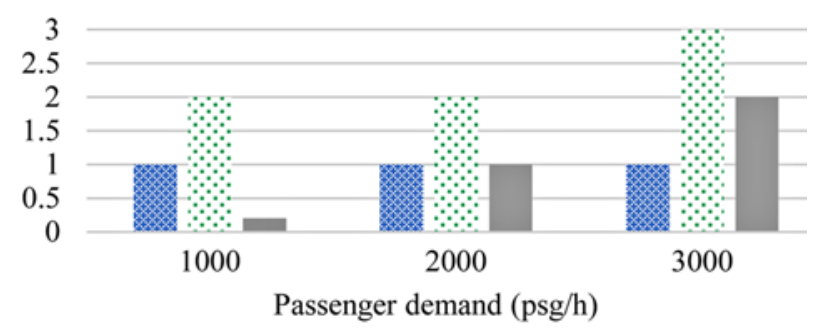

(a)

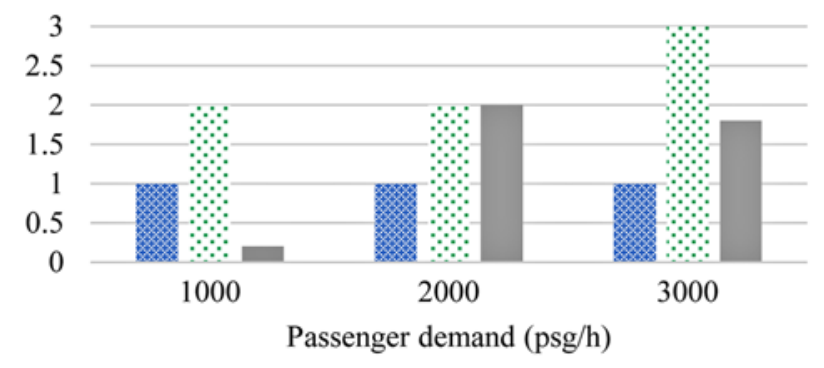

(b)

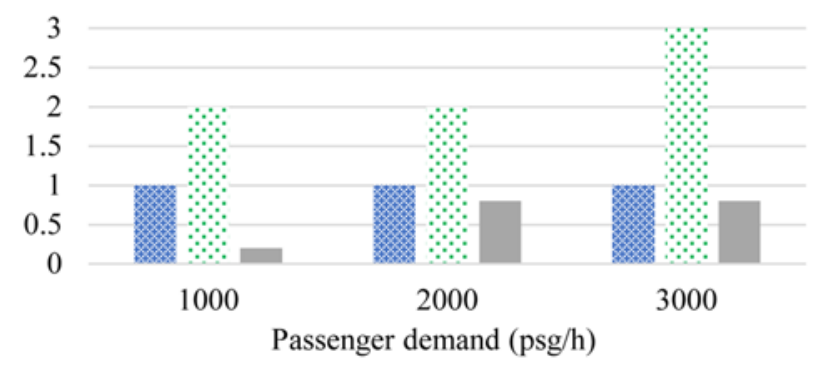

(c)

絭MFEL $\because$ HOV critical occupancy $\approx$ LOV price rate

Fig. 8. MFEL Design and Management under Varying Passenger Demand on 2-Lane Road: (a) Emergency $=0 \mathrm{veh} / \mathrm{h}$, (b) Emergency $=$ $1 \mathrm{veh} / \mathrm{h}$, (c) Emergency $=2 \mathrm{veh} / \mathrm{h}$

generally allow lower HOV critical occupancy, while corresponds to increased LOV price rate when passenger demand is high up to $4,000 \mathrm{psg} / \mathrm{h}$. Thus it comes to the conclusion that the road with more lanes can promote consistent management rule for both HOV and LOV, while 2-lane roads are sensitive to passenger demand to increase HOV critical occupancy and LOV price rate to enhance road utility and to accommodate more passengers.

\subsubsection{Effect of Bus Occupancy}

Taking the road with 3 lanes for example, Fig. 10 shows the proposed scheme from the established model with bus occupancy ranging between 30 and $80 \mathrm{psg} / \mathrm{veh}$. It is observed that MFEL design and HOV critical occupancy are generally consistent across different bus occupancy, but not LOV price rate. For example, with bus occupancy being $30 \mathrm{psg} / \mathrm{veh}$, LOV price rate jumps to 1.6 RMB/km when passenger demand increases from 3,000 to $4,000 \mathrm{psg} / \mathrm{h}$, where non-MFEL traffic is close to saturation, making MFEL competitive and costly. In comparison, when bus occupancy increases to $60 \mathrm{psg} / \mathrm{veh}$ or more, LOV price rate just increases to $1.2 \mathrm{RMB} / \mathrm{km}$ when passenger demand is $4,000 \mathrm{psg} / \mathrm{h}$, as higher bus occupancy manages to help reduce traffic saturation, resulting in

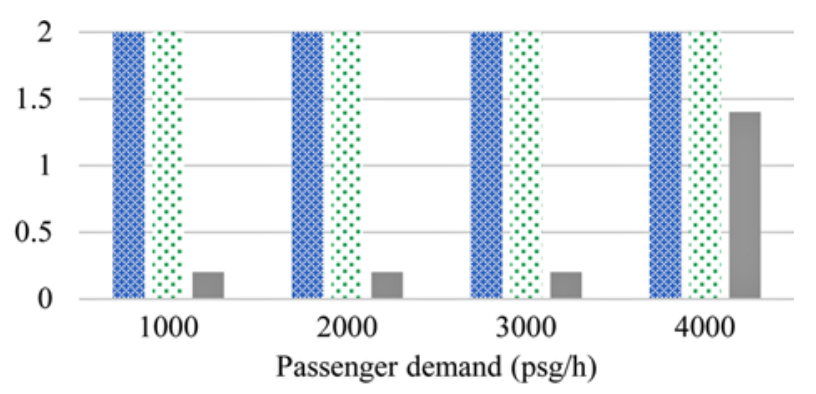

(a)

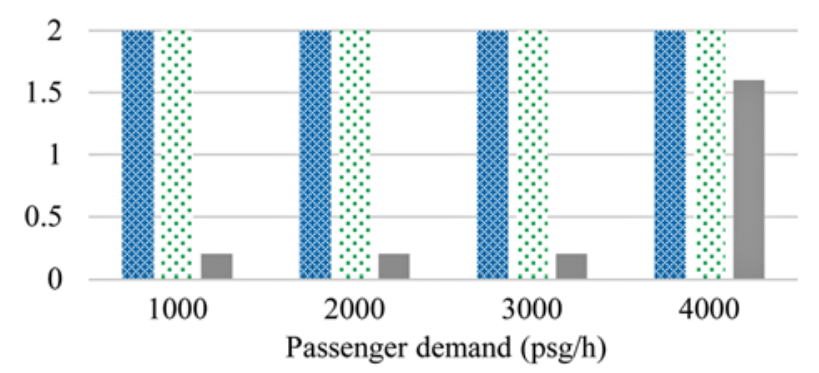

(b)

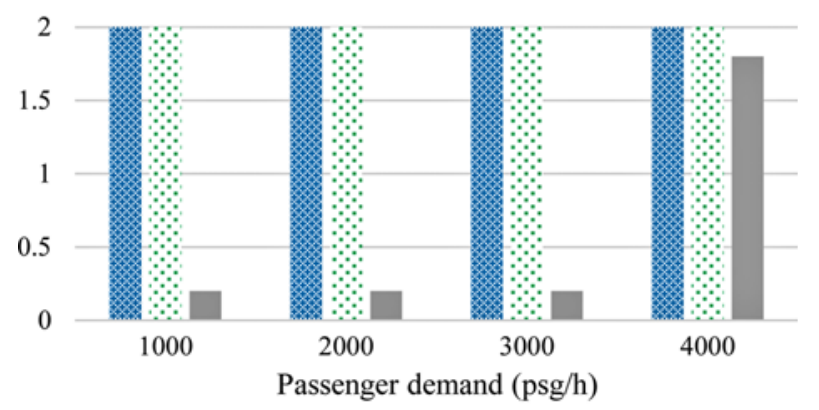

(c)

MFL $\because$ HOV critical occupancy $=$ LOV price rate

Fig. 9. MFEL Design and Management under Varying Passenger Demand on 3-Lane Road: (a) Emergency $=0 \mathrm{veh} / \mathrm{h}$, (b) Emergency $=$ $1 \mathrm{veh} / \mathrm{h}$, (c) Emergency $=2 \mathrm{veh} / \mathrm{h}$

friendly LOV pricing. Thus it comes to the conclusion that higher bus occupancy contributes to enhanced road utility, and promotes modest LOV pricing regardless of passenger demand levels.

\section{Conclusions}

MFEL, competing EBL and HOV lane with higher utility rate and promoting personalized trip choices, provides priority to traffic modes including emergency vehicles, buses, HOVs, and priced LOVs. Considering that these modes are characterized with distinct operation pattern and different management strategy, it is necessary to explore the possibility to comprehensively serve these prioritized trip modes on the same lane. That is, emergency vehicles, buses, HOVs, and priced LOVs are combined for the optimal MFEL design and management against site-specific traffic conditions. Targeting at the four major types of traffic delay (i.e., lane access, bus stop dwelling, signalized intersections, 


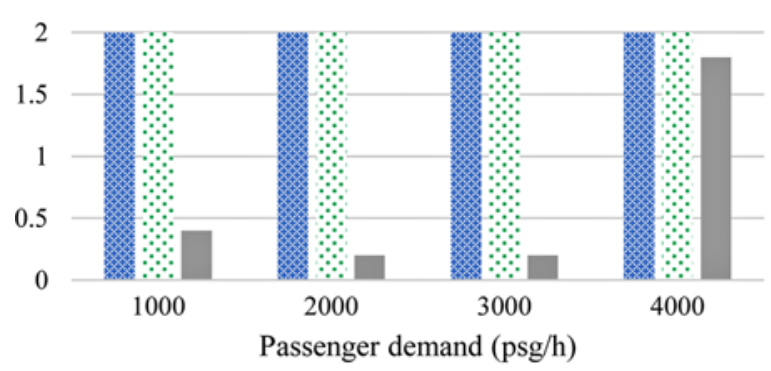

(a)

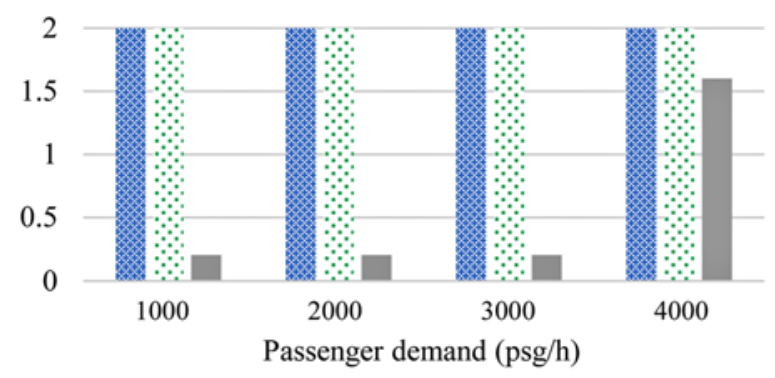

(b)

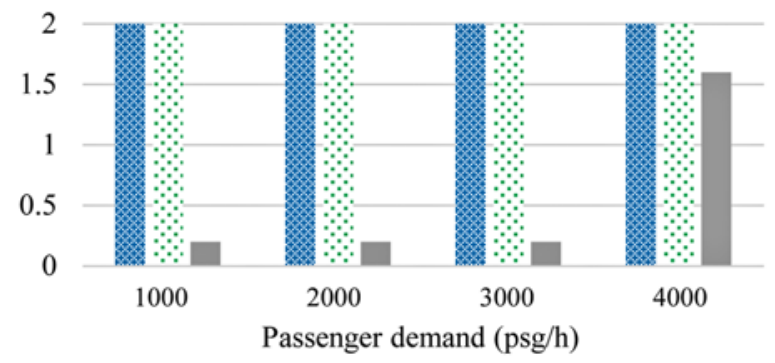

(c)

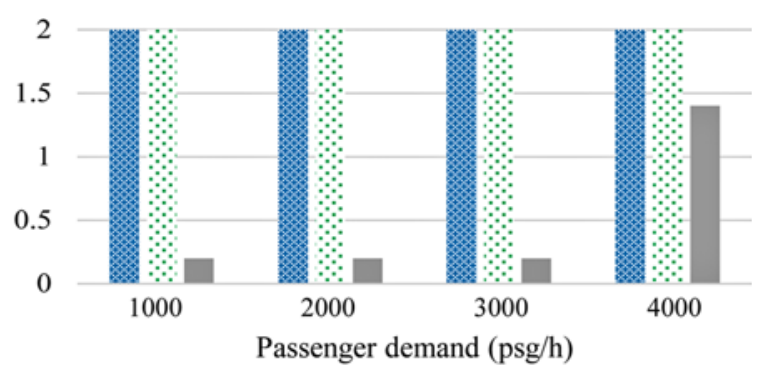

(d)

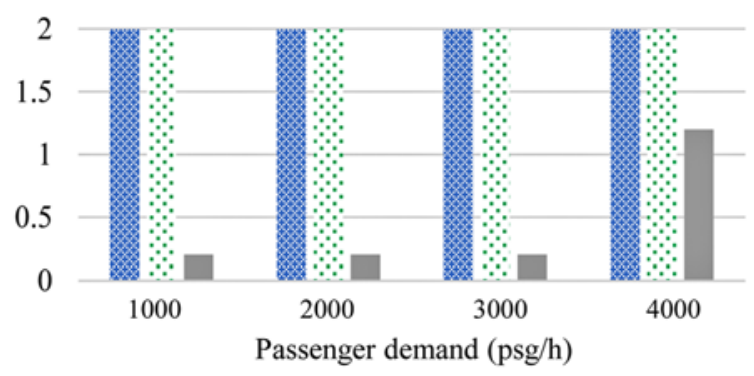

(e)

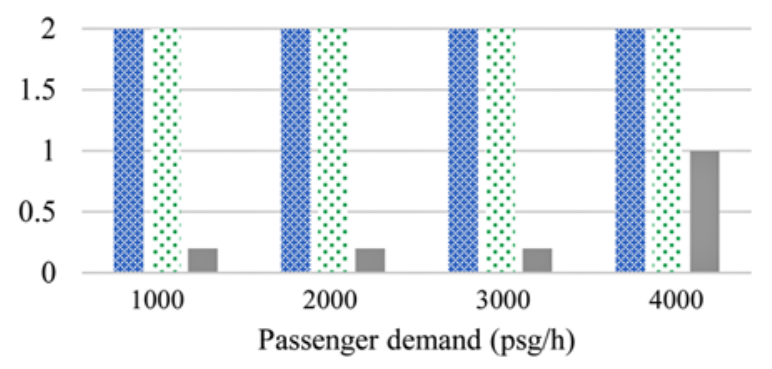

(f)

MFL $\because$ HOV critical occupancy $\quad$ LOV price rate

Fig. 10. MFEL Design and Management with Varying Bus Occupancy: (a) Bus Occupancy = 30 psg/veh, (b) Bus Occupancy = 40 psg/veh, (c) Bus Occupancy $=50$ psg/veh, (d) Bus Occupancy $=60$ psg/veh, (e) Bus Occupancy $=70$ psg/veh, (f) Bus Occupancy $=80$ psg/veh

and ride-sharing) and four kinds of trip cost, total trip utility is calculated, based on which modal choice is made among buses, HOVs, priced LOVs, and GVs. Trip time of different vehicle types are accordingly weighted to reflect their importance, where emergency vehicles benefit most, followed by buses, HOVs, and LOVs. Enumeration algorithm is then developed to solve this model for the optimal MFEL design and management.

Case study follows to calibrate and validate the proposed MFEL scheme on the 4-lane road under varying passenger demand and emergency vehicle frequency. It is found that the proposed scheme recommends MFEL adoption regardless of traffic demand, which reduces total delay especially when traffic is nearly saturated. Sensitivity analyses are conducted to test the effect on MFEL of road lane count and bus occupancy, finding that more lanes on the road provides the flexibility to accommodate increased passenger demand without changing MFEL management. Moreover, higher bus occupancy assists in avoiding significant increase of LOV price rate, to promote stable and friendly pricing for trip equality. This research is an extension from the existing literature with systematic framework that determines trip share with comprehensive utility and modal choice, instead of predetermining bus frequency or HOV critical occupancy.

Major limitation of the proposed research is four-fold. First, although this research explores the joint design and management of MFEL that serves vehicles with multiple priority levels, it does not establish the priority method against dynamic and stochastic traffic demand. This is critical to securing MFEL efficiency on the urban corridor and helps to develop the concrete stakes that prioritized vehicles may take advantage of. Second, the speed difference of vehicles moving on MFEL and GPL should be carefully addressed, to smooth their conflicts and secure safety without affecting traffic efficiency. For example, the location of MFEL access and exits needs delicate design to address the weaving of vehicles moving onto and out of MFEL, delay from which should be carefully modeled instead of being constant as assumed in the current research. Third, travelers' heterogeneous willingness of paying or carpooling on MFEL and flexibility in changing departure time in response to MFEL management rule is not considered in the current model, which influences trip mode choice and is fundamental to MFEL success 
in the practice. Forth, the temporal shift of the managerial rules of HOV critical occupancy and LOV paying rate requires more efforts, so as to respond to the changing travel demand and to maintain MFEL attraction as well as utility. Stochasticity or uncertainty of trip demand and modal choice as well as their impact on MFEL design/management should also be addressed in future study (Tirkolaee and Aydın, 2021; Tirkolaee et al., 2021).

On-going research focuses on the integrated design of MFEL access and exit, so as to enhance MFEL accessibility and utility without causing safety concern or management difficulty.

\section{Acknowledgments}

This work has been supported by the National Natural Science Foundation of China (Grant No. 52002261) and China postdoctoral science foundation (Grant No. 2020M671581).

\section{ORCID}

Zhiquan Xie ๑ https://orcid.org/0000-0003-3115-1760

Hui Jin ๑ https://orcid.org/0000-0003-1233-8466

Jing Teng ๑ https://orcid.org/0000-0003-2130-0208

Xiaoguang Yang ๑ https://orcid.org/0000-0002-1121-9011

\section{References}

Abdelfatah A, Abdulwahid AR (2017) Impact of exclusive bus lanes on traffic performance in urban areas. Proceedings of the 2nd world congress on civil, structural, and environmental engineering (CSEE'17), April 2-4, Barcelona, Spain

Akcelik R (1988) The highway capacity manual delay formula for signalized intersections. ITE Journal 58:3(3):23-27

Boudhrioua S, Shatanawi M (2019) Implementation of absolute priority in a predictive traffic actuation schemes. Periodica Polytechnica Transportation Engineering 49(2)

Brown B, Aicp LB (2008) High-occupancy-vehicle user survey: Washington State freeway HOV system. Proceedings of transportation research board 87th annual meeting, January 13-17, Washington DC, USA

Burris MW, Stockton BR (2004) Hot lanes in Houston - Six years of experience. Journal of Public Transportation 7(3):1-22, DOI: 10.5038/2375-0901.7.3.1

Esfeh MA, Wirasinghe SC, Saidi S, Kattan L (2020) Waiting time and headway modelling for urban transit systems - A critical review and proposed approach. Transport Reviews 41(2):141-163, DOI: 10.1080/ 01441647.2020.1806942

Jang K, Song MK, Choi K, Kim DK (2014) A bi-level framework for pricing of high-occupancy toll lanes. Transport 29(3):317-325, DOI: $10.3846 / 16484142.2014 .952248$

Kwon J, Varaiya P (2008) Effectiveness of California's high occupancy vehicle (HOV) system. Transportation Research Part C: Emerging Technologies 16(1):98-115, DOI: 10.1016/j.trc.2007.06.008

Laval JA, Cho HW, Muñoz JC, Yin Y (2015) Real-time congestion pricing strategies for toll facilities. Transportation Research Part B 71:19-31, DOI: 10.1016/j.trb.2014.09.015

Liu HY, Wang J, Wijayaratna K, Dixit VV, Waller ST (2015) Integrating the bus vehicle class into the cell transmission model. IEEE Transactions on Intelligent Transportation Systems 16(5):26202630, DOI: 10.1109/TITS.2015.2413995

Lombardi C, Picado-Santos L, Annaswamy AM (2021) Model-based dynamic toll pricing: An overview. Applied Sciences 11(11):4778, DOI: 10.3390/APP11114778

Nellore K, Hancke GP, Bellavista P (2016) Traffic management for emergency vehicle priority based on visual sensing. Sensors 16(11): 1892, DOI: $10.3390 / \mathrm{s} 16111892$

Newmark GL (2014) HOT for transit? Transit's experience of highoccupancy toll lanes. Journal of Public Transportation 17(3):97114, DOI: 10.5038/2375-0901.17.3.7

Nuzzolo A, Comi A (2016) Individual utility-based path suggestions in transit trip planners. IET Intelligent Transport Systems 10(4):219226, DOI: 10.1049/iet-its.2015.0138

Olstam J, Häll CH, Smith G (2015) Dynamic bus lanes in Sweden - A pre-study. K2 Research, DOI: 10.13140/RG.2.1.2943.9127

Pessaro B, Turnbull KF, Zimmerman C (2013) Impacts to transit from variably priced toll lanes: Results from U.S. Department of Transportation Urban Partnership Agreements. Transportation Research Record 2396(1):117-123, DOI: 10.3141/2396-13

Poole R (2020) The impact of HOV and HOT lanes on congestion in the United States. Proceedings of the international transport forum discussion papers, September 21, Tallinn, Estonia

Rossi ML (2019) Congestion mitigation and student mode choice: A statistical analysis of the I-66 high occupancy toll lanes impact on transit and university student mode choice in the Washington DC area. MSc Thesis, George Mason University, Fairfax, VA, USA

Saberi M, Figliozzi MA (2011) A study of freeway volume-to-capacity ratio based travel time approximations using archived loop detector data. Proceedings of the 90th annual meeting of the transportation research board, January 23-27, Washington DC, USA

Tan Z, Gao HO (2018) Hybrid model predictive control based dynamic pricing of managed lanes with multiple accesses. Transportation Research Part B: Methodological 112:113-131, DOI: 10.1016/ j.trb.2018.03.008

TCRP (2013) Transit capacity and quality of service manual, third edition. Transit Cooperative Research Program Document 165, Transportation Research Board, Washington DC, USA

Tirkolaee EB, Abbasian P, Weber GW (2021) Sustainable fuzzy multitrip location-routing problem for medical waste management during the COVID-19 outbreak. Science of the Total Environment 756:143607, DOI: 10.1016/j.scitotenv.2020.143607

Tirkolaee EB, Aydın NS (2021) A sustainable medical waste collection and transportation model for pandemics. Waste Management \& Research 39(1):34-44, DOI: 10.1177/0734242X211000437

Tirkolaee EB, Hadian S (2019) A robust green traffic-based routing problem for perishable products distribution. Computational Intelligence 36(1):80-101, DOI: 10.1111/coin.12240

Wu W, Head L, Yan S (2018) Development and evaluation of bus lanes with intermittent and dynamic priority in connected vehicle environment. Journal of Intelligent Transportation Systems 22(4):301-310, DOI: 10.1080/15472450.2017.1313704

Wu P, Liu S, Yang C (2017) A stochastic programming model for optimal bus lane reservation under uncertainty. Proceedings of the European conference on stochastic optimization, September, Rome, Italy

Xiao LL, Liu TL, Huang HJ (2016) On the morning commute problem with carpooling behavior under parking space constraint. Transportation Research Part B: Methodological 91:383-407, DOI: 10.1016/ j.trb.2016.05.014 
Xiao LL, Liu TL, Huang HJ (2019) Tradable permit schemes for managing morning commute with carpool under parking space constraint Transportation 48:1563-1586, DOI: 10.1007/s11116-019-09982-w

Xu F, Du Y, Sun L (2013) A framework for ongoing performance monitoring of bus lane system. Procedia-Social and Behavioral Sciences 96:175-181, DOI: 10.1016/j.sbspro.2013.08.023

Yang H (1998) When should carpool lanes be introduced in a multi-lane highway? Journal of Advanced Transportation 32(2):242-252, DOI: 10.1002/atr.5670320207
Zang G (2021) Entrance location planning of HOV lanes in multi-lane highway considering system and HOV lane state. Journal of Physics: Conference Series 1910(1), DOI: 10.1088/1742-6596/1910/1/012014

Zhou G, Mao L, Hu P, Zhou Z (2020) Research on HOV lane priority dynamic control under connected vehicle environment. Journal of Advanced Transportation 2020(4):1-12, DOI: 10.1155/2020/8892859

Zitzow-Childs SB (2017) A corridor-based methodology for the design of open access high occupancy toll lane facilities. MSc Thesis, University of Minnesota, Minneapolis, MN, USA 